\title{
Proceso de arbitraje para la calidad de las revistas científicas
}

\author{
Peer review process to ensure the quality of scientific journals
}

\author{
María del Carmen López Jordi ORCID: 0000-0002-9025-3188 \\ Sylvia Piovesan ${ }^{1}$ ORCID: 0000-0003-1807-6380 \\ Vanesa Pereira-Prado ${ }^{1}$ ORCID: 0000-0001-7747-671 \\ Carina Patrón ${ }^{1}$ ORCID: 0000-0002-8662-9437 \\ Anahí Lima ${ }^{1}$ ORCID: 0000-0003-2638-8210
}

\section{Resumen}

La revisión por pares representa uno de los pilares más importantes sobre los que se sustentan las revistas científicas. Es un proceso complejo y riguroso realizado por expertos nacionales y/o extranjeros que permite la evaluación crítica de los manuscritos. En el área salud, este proceso toma una dimensión relevante sobre todo en la valoración de la credibilidad de los resultados de investigaciones y en la descripción de procedimientos y resolución de casos.

Palabras clave: revisión por pares, evaluación de expertos, proceso arbitral. 


\section{Abstract}

The peer-review process is one of the main pillars of scientific journals. It is a complex and rigorous activity conducted by national or foreign experts, which allows for a critical assessment of manuscripts. In the health area, this process becomes highly relevant, especially regarding assessing the credibility of the research results and the description of procedures and resolution of cases.

Keywords: peer review, expert evaluation, arbitration process.

\section{Particularidades de la revisión por pares}

La revisión de los artículos por pares representa uno de los pilares más importantes sobre los que se sustentan las publicaciones científicas como el caso de Odontoestomatología. Es un proceso complejo y riguroso realizado por expertos nacionales y/o extranjeros que permite la evaluación crítica de los manuscritos recibidos para publicación en una revista científica. En el área salud este proceso toma una dimensión relevante sobre todo en la valoración de la credibilidad de los resultados de investigaciones y en la descripción de procedimientos y resolución de casos.

La revisión por pares o peer review tiene ventajas para todos los involucrados en el proceso editorial, esto es: los editores porque la evaluación experta colabora en la decisión editorial sobre los manuscritos y los autores dado que las sugerencias, ajustes y correcciones de los árbitros les permite mejorar el artículo, optimizando su calidad ${ }^{(1)}$.

La selección adecuada de los árbitros, de acuerdo a la temática del manuscrito, es una de las principales tareas del equipo editorial de las re-

\section{Resumo}

De avaliação pelos pares representa um dos pilares mais importantes no qual estão baseados os periódicos científicos de avaliação pelos pares. É um processo complexo e rigoroso conduzido por peritos nacionais ou estrangeiros, que permite uma avaliação crítica dos manuscritos. $\mathrm{Na}$ área da saúde, este processo leva acima de tudo uma dimensão relevante na avaliação da credibilidade dos resultados da pesquisa e na descrição de procedimentos e resolução de casos.

Palavras chave: revisão por pares, avaliação especializada, proceso de arbitragem.

vistas científicas y también el control sistemático en el cumplimiento de las etapas involucradas, por ejemplo, cuando el árbitro solicita una nueva revisión luego de los ajustes de los autores, cuando los dos árbitros emiten informes contradictorios (uno acepta y otro rechaza un artículo), por lo que debe seleccionarse un revisor adicional para seguridad en la resolución, etc.

\section{Tipos de proceso de revisión}

La revisión por pares se puede realizar de tres formas: a) simple-ciega, b) doble ciega y c) abierta $^{(2)}$.

a) En la revisión simple-ciego, el revisor conoce la identidad del autor pero el autor no conoce la del revisor; es una práctica actualmente aceptada

b) En la revisión por par doble ciego, tanto los revisores como los autores son anónimos; es la forma más aceptada actualmente dado que no se informa sobre la identidad de autores o revisores. Con este enfoque se busca preservar el anonimato, asegurando así que la revisión se haga de forma justa. Sin embargo, en un área pequeña como el Uruguay, es difícil ocultar la 
identidad de un autor por lo que en general los árbitros seleccionados para evaluar un artículo nacional son extranjeros.

c) La revisión por pares abierta revela las identidades de ambos, autores y revisores, y los autores tienen la capacidad de identificar los comentarios de los revisores ${ }^{(3)}$.

El árbitro además de aportar mejoras al manuscrito cuando guía, comenta y sugiere al autor basado en sus propios conocimientos, contribuye con el editor a detectar malas prácticas o conductas no éticas en el sentido de detectar plagio y fraude en la publicación científica ${ }^{(4)}$.

Carácter de la revisión por pares. El carácter anónimo de los revisores es una práctica general, aunque no es necesariamente aceptada por todos los expertos. Por un lado el equipo editor puede recibir el reclamo de un árbitro para ser informado de los nombres y/o filiaciones institucionales de los autores, esto sucede muy raramente. De hecho, un buen árbitro, conocedor de su campo y de los investigadores que en él trabajan, podría llegar a detectar el origen de un trabajo, al menos a nivel de grupo de investigación, sin que la información específica le haya sido proporcionada. Por lo mencionado, puede llegar a darse en alguna ocasión que especialistas a quienes se les solicita un arbitraje declinen hacerlo, con el argumento de conocer a alguno de los autores o de tener conflictos de interés.

En un sistema anónimo, a los autores no se les revela la identidad de los árbitros que evalúan sus trabajos a fin de evitar de esa manera conflictos y posibles enfrentamientos.

El tiempo y el esfuerzo generosamente cedidos por los árbitros, sólo se ven compensados por la satisfacción de haber sido seleccionados para la evaluación del manuscrito que surge de un reconocimiento de su experticia en la temática. A modo de actuar con transparencia es una buena práctica por parte de las publicaciones científicas poner en conocimiento público y periódicamente la identidad de los revisores que han contribuido en ese lapso de tiempo.
En este sentido, a continuación se presenta la nómina de revisores de Odontoestomatología que actuaron en los años 2018 y 2019. En este período fueron publicados 40 artículos que comprenden 20 trabajos nacionales y 20 extranjeros, siendo 10 los manuscritos no aceptados.

\begin{tabular}{|c|c|c|c|c|c|}
\hline \multicolumn{6}{|c|}{ № ÁRBITROS QUE PARTICIPARON TOTAL } \\
\hline & & & Nacionales & \multicolumn{2}{|c|}{ Extranjeros } \\
\hline & \multirow{2}{*}{\multicolumn{2}{|c|}{$\begin{array}{l}\text { Año } 2018 \\
\text { Año } 2019\end{array}$}} & 18 & \multicolumn{2}{|c|}{30} \\
\hline & & & & \multicolumn{2}{|c|}{13} \\
\hline \multicolumn{3}{|c|}{ TOTAL } & 25 & \multicolumn{2}{|c|}{43} \\
\hline \multicolumn{6}{|c|}{ PROCEDENCIA DE ÁRBITROS EXTRANJEROS } \\
\hline & Argentina & Brasil & Chile & México & Perú \\
\hline 2018- & 17 & 4 & 7 & 1 & 1 \\
\hline 2019 & 9 & 2 & 1 & 0 & 1 \\
\hline TOTAL & 26 & 6 & 8 & 1 & 2 \\
\hline
\end{tabular}

Nómina de árbitros 2018-2019

\begin{tabular}{|c|c|c|}
\hline 1. & Alcántara, Raúl & Argentina \\
\hline 2. & Álvarez, Licet & Uruguay \\
\hline 3. & Ángeles Medina, Fernando & México \\
\hline 4. & Arriagada, María & Chile \\
\hline 5. & Artaza, Liliana & Argentina \\
\hline 6. & Barone, Dante & Brasil \\
\hline 7. & Bella, Marcela & Argentina \\
\hline 8. & Borgia, Ernesto & Uruguay \\
\hline 9. & Bueno, Luis & Uruguay \\
\hline & Cançado, Marcia & Brasil \\
\hline & Cantero, Cecilia & Chile \\
\hline & Casamayou, Martha & Uruguay \\
\hline & Coco, Laura & Argentina \\
\hline & Cornejo, Susana & Argentina \\
\hline & Cosseti, Laura & Uruguay \\
\hline & Di Nasso, Patricia & Chile \\
\hline & Fernández, Claudia & Argentina \\
\hline & Funosas, Esteban & Argentina \\
\hline & Garrofé, Analía & Argentina \\
\hline
\end{tabular}




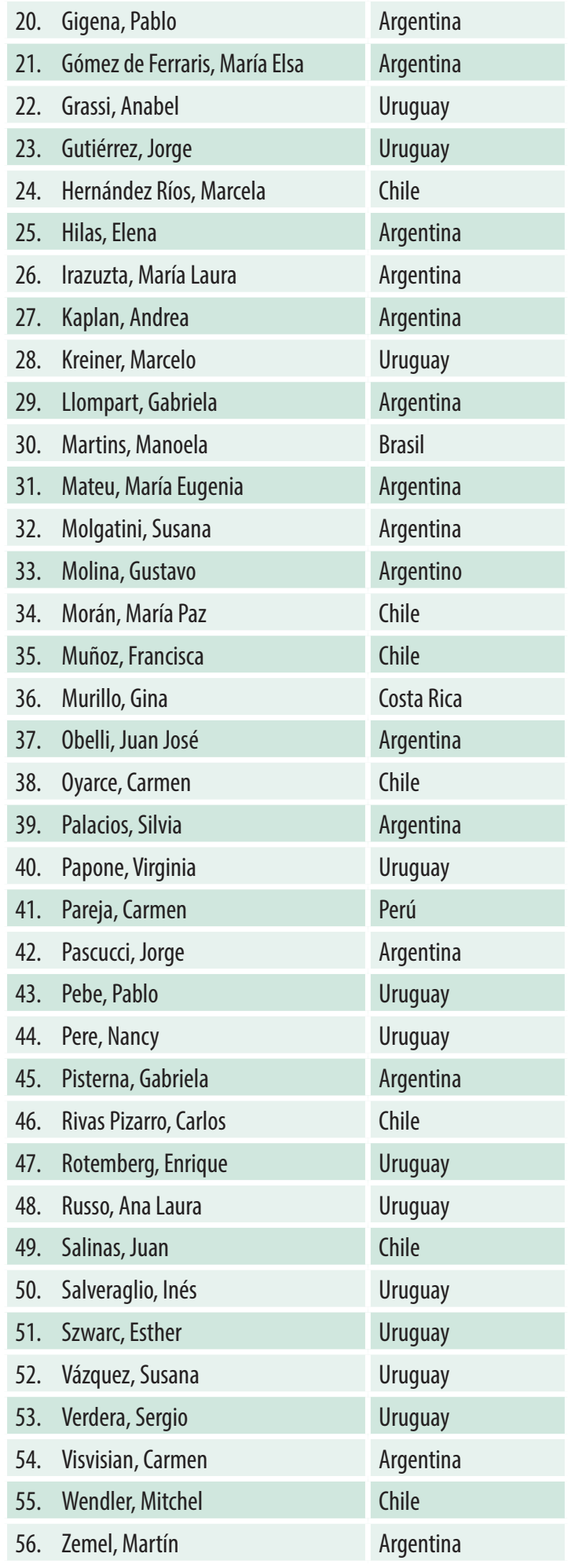

\section{Contribución de autoría}

1. Concepción y diseño del estudio

2. Adquisición de datos

3. Análisis de datos

4. Discusión de los resultados

5. Redacción del manuscrito

6. Aprobación de la versión final del manuscrito

MCLJ ha contribuido en: 1, 2, 3, 4, 5 y 6 . SP ha contribuido en: 1, 2, 3, 4, 5 y 6 .

VP ha contribuido en: 2,3 y 6 .

CP ha contribuido en: 2,3 y 6 .

AL ha contribuido en: 2,3 y 6 .

\section{Referencias}

1. Ladrón de Guerrera M, Hincapié J, Jaclman J, Herrera O, Caballero CV. Revisión por pares, ¿Qué es y para qué sirve? Rev Cien Salud Uninorte 2008;24(2).

2. Nassi-Calo, L. Revisión por pares: modalidades, pros y contras [online]. SciELO en Perspectiva, 2015. Disponible en: https://blog.scielo.org/ es/2015/03/27/revision-por-pares-modalidades-pros-y-contras/

3. SciELO Guidelines: The Peer Review Process in Journal Publishing. Version September 2018.Disponible en: https://docs.google.com/ document/d/1iTmddgQqqbZGqBLHst1r40m owkCsAHEpKcNhtYIGnW0/edit?usp=sharing

4. Committee on Publication Ethics -COPE. Disponible en: https://publicationethics.org/se $\operatorname{arch}$ ?t=plagiarism \& type $\% 5 \mathrm{~B} \% 5 \mathrm{D}=$ Guidelines \&sort=score

María del Carmen López Jordi: dra.lopezjordi@gmail.com 